\title{
AceCloud: Molecular Dynamics Simulations in the Cloud
}

\author{
M. J. Harvey*,† and G. De. Fabritiis ${ }^{*, \ddagger}$ \\ Acellera Ltd, London, UK, and Universitat Pompeu Fabra, Barcelona, Spain and Institució \\ Catalana de Recerca i Estudis Avançats, Passeig Lluis Companys 23, 08010 Barcelona, \\ Spain \\ E-mail: m.j.harvey@acellera.com; gianni.defabritiis@upf.edu
}

\begin{abstract}
We present AceCloud, an on-demand service for molecular dynamics simulations. AceCloud is designed to facilitate the secure execution of large ensembles of simulations on an external cloud computing service (currently Amazon Web Services).

The AceCloud client, integrated into the ACEMD molecular dynamics package, provides an easy-to-use interface that abstracts all aspects of interaction with the cloud services. This gives the user the experience that all simulations are running on their local machine, minimising the learning curve typically associated with the transition to using high performance computing services.
\end{abstract}

\footnotetext{
${ }^{*}$ To whom correspondence should be addressed

$\dagger$ Acellera

$\ddagger \mathrm{UPF} / \mathrm{ICREA}$
} 


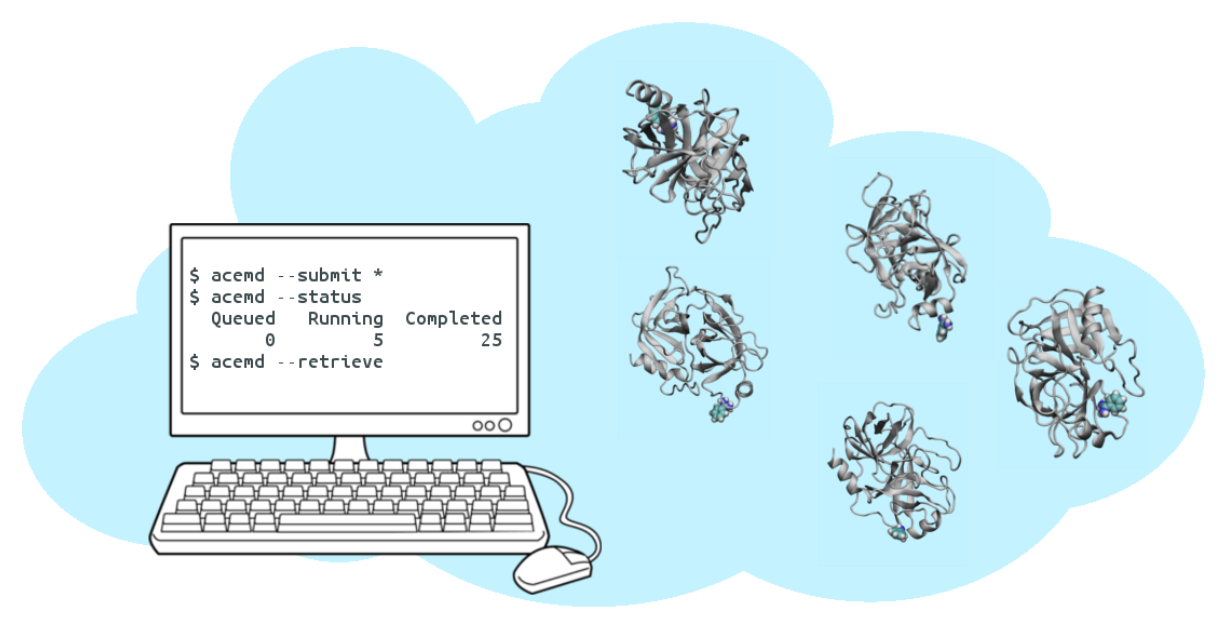

\section{Introduction}

In recent years classical molecular dynamics simulation has begun to prove an increasingly powerful complement to experiment, as the timescales accessible through simulation approach biologically-relevant timescales.

These developments are underpinned by progress in both simulation technology and methodology. In particular, the exploitation of specialised accelerator processors (known as GPUs, belying their origin in the graphics processing field) has proven transformative for the field. A modern GPU-based MD code - such as ACEMD, ${ }^{1}$ GROMACS $^{2}$ or Amber ${ }^{3}-$ is able to simulation rates on a single low-cost GPU that may otherwise require several nodes of a dedicated high performance compute cluster. This effective decoupling of MD from the established HPC space has led to substantial (over an order of magnitude) reduction in cost per unit of MD sampling.

This marked reduction in cost of MD sampling, yet without substantially increasing the timescales accessible within any one simulation, has prompted the development of methods based upon the analysis of ensembles of large numbers of independent simulations. Markov modelling techniques, for example, have been demonstrated to be able to recover equilibrium properties and kinetic processes that occur over timescales far longer than that of any one simulation. ${ }^{4}$ Such studies are computationally-expensive and special-purpose infrastruc- 
tures, for example GPUGRID ${ }^{5}$ and Folding@Home,${ }^{6}$ have been constructed to support these activities by exploiting GPU computing resources provided by volunteers.

Recourse to unconventional sources of compute resource has been necessary because, despite GPUs now well-established as a significant part of the contemporary HPC landscape they have limited penetration in established HPC facilities. ${ }^{7}$ In large part, this is attributable to the need for software to be substantially refactored in order to run on a GPU. On a mixedworkload system where only a faction of capacity is consumed by GPU-capable software, or in the absence of funding for software development, for example, it can be difficult to justify the cost of deploying GPUs. This has important implications for the broader uptake of GPU-dependent ensemble-MD-based analytical methods: evaluation of these techniques is limited to groups that already access to substantial GPU resources.

To address this problem we have developed AceCloud, a service that provides online, on-demand access to compute resources for molecular dynamics simulation. AceCloud takes advantage of the broader computing industry trend of 'cloud' computing ${ }^{8}$ - effectively the flexible rental of computing resources - to lower the barrier to entry for performing largescale molecular dynamics simulations. The capital setup costs of establishing in-house GPU resources are negated and replaced by on-going operational costs that are proportional to usage.

AceCloud natively supports the popular molecular dynamics codes ACEMD and GROMACS, as well as permitting the easy execution of other third-party software, such as Amber or NAMD. The current implementation of AceCloud targets the Amazon Web Services (AWS) ${ }^{9}$ Cloud computing platform.

To minimise the learning curve associated with cloud computing, AceCloud is accessed through simple extensions to the ACEMD molecular dynamics code. This interface abstracts all interaction with the supporting cloud infrastructure, and emulates, as far as is practicable, the experience of running simulations locally on one's own machine. This focus on ease-ofuse minimise the need for the user to change or adapt existing working practices, a burden 
often encountered when transitioning from personal desktop to HPC computing systems.

In this manuscript we present the design and implementation of AceCloud along with examples of use.

\section{Design and Implementation}

AceCloud is designed to execute and manage large ensembles of molecular dynamics simulations on third party cloud resources. The user experience is intended to closely replicate that of command-line program execution, without exposing the details of interaction with the cloud service. All cloud operations (data transfer, virtual machine instantiation, etc) are mediated by the client, giving the appearance of completely local execution.

Currently, AceCloud can target Amazon Web Services. AWS is presently (Jan 2015) the only major cloud provider that makes available GPU-based compute resources.

The following sections detail the user client and user experience, and overview of the AWS features used by AceCloud, and the implementation of the cloud-side compute service.

\section{Client Software and User Experience}

The AceCloud client is implemented as an extension to the ACEMD molecular dynamics simulation code. ACEMD has a command-line interface, with new AceCloud functions being accessed via a new set of command-line switches (Table 1). The default behaviour of ACEMD is to perform a molecular dynamics simulation on the machine on which it is being run, starting from appropriate input files found in the current working directory. The addition of the command line argument --submit is sufficient to cause ACEMD to instead submit the job for execution on AceCloud (Figure 1 shows an example).

Whereas local execution is synchronous, with control not returning to the user until the simulation is completed, AceCloud jobs are asynchronous. Control is immediately returned to the user after submission, and additional commands are used to monitor job status and 
results for finished simulations back to the directories from which they were originally created. Results of finished simulations are stored by AceCloud (in S3) until the user performs a retrieval and automatically deleted afterwards.

For long-running simulations, partial results can be obtained using the additional argument --incremental. This instructs the client to use SSH and rsync to synchronise the local copy of the output with the current progress of the simulation. rsync is used to ensure that successive retrievals only copy new or changed data, so minimising network bandwidth usage.

Running a retrieval as a non-terminating background process, with the options --incremental, provides the user experience most similar to having the simulations running locally. Output appears incrementally in the job directories soon after its creation, without any further user interaction with AceCloud being necessary.

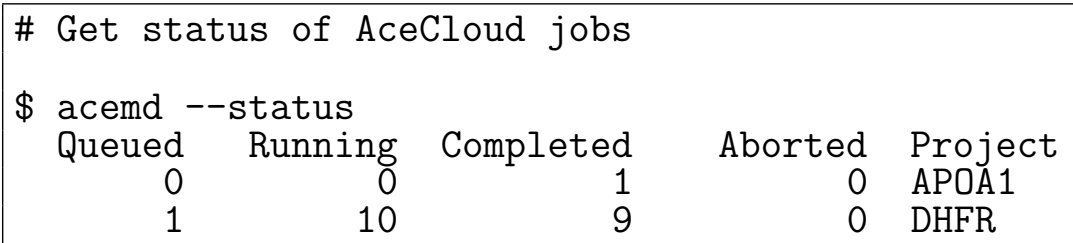

Figure 2: An example of querying the status of AceCloud jobs. In this instance, 21 jobs are extant, grouped into two projects, APOA1 and DHFR. The DHFR project contains 20 jobs, 10 in progress, 9 completed and 1 pended to run.

AceCloud has built-in support for ACEMD and GROMACS simulations, and support for running arbitrary third-party code (for example Amber or NAMD). When instantiated, the AceCloud VM will determine whether to run ACEMD or GROMACS based on the presence of specific files ( input for ACEMD, topol.tpr for GROMACS). If neither of these are present, a file called run. sh will be executed. This provides a mechanism by which the user may run arbitrary code on an AceCloud instance.

The MD codes Amber and NAMD, for example, are not included within AceCloud because of license restrictions. A user in possession of a suitable license may run their own 
Table 1: AceCloud client command line arguments

\begin{tabular}{|c|c|}
\hline Argument & Description \\
\hline --command [command] & $\begin{array}{l}\text { Give syntax and description of an ACEMD input file } \\
\text { command. }\end{array}$ \\
\hline --device [dev1ist] [inputfile] & $\begin{array}{l}\text { Run a simulation on the specified comma-separated list } \\
\text { of GPUs. If no input file name is specified, input is } \\
\text { assumed. }\end{array}$ \\
\hline --subnit [[--project prj-nane] directory...] & $\begin{array}{l}\text { Run jobs on Amazon EC2. Submits each directory to } \\
\text { Amazon as a separate simulation. Each simulation is } \\
\text { allocated to the project prj-name that precedes it in the } \\
\text { argument list. If no directory is specified, the current } \\
\text { working directory will be used. }\end{array}$ \\
\hline --status $[$ prj-name...] & $\begin{array}{l}\text { Return the status of all jobs in project summarised by } \\
\text { project, or all jobs in the project if --long is specified. }\end{array}$ \\
\hline- -retrieve $[$ prj-name....] [--1oop] [--incremental] & $\begin{array}{l}\text { Retrieve the results of all completed simulations, or only } \\
\text { those in the specified projects. Results are placed in } \\
\text { the originating directories. Makes a single pass then } \\
\text { exits unless --loop is specified. Add --incremental to } \\
\text { retrieve partial results from running simulations. }\end{array}$ \\
\hline --delete [--project prj-name] [dir-1ist] & $\begin{array}{l}\text { Delete simulations and pending output of the specified } \\
\text { project. If dir-list is specified only those named simula- } \\
\text { tions will be deleted. If no dir-list everything under the } \\
\text { project prj-name will be deleted. }\end{array}$ \\
\hline --spots & Show current Amazon Spot pricing \\
\hline --configure & $\begin{array}{l}\text { Set Amazon configuration options. --configure help } \\
\text { for info. }\end{array}$ \\
\hline --verbose & $\begin{array}{l}\text { Add as a qualifier to the above to produce verbose out- } \\
\text { put. }\end{array}$ \\
\hline$--\mathrm{xm} 1$ & $\begin{array}{l}\text { Add as a qualifier to the above to produce structured } \\
\text { output. }\end{array}$ \\
\hline
\end{tabular}


copy by placing the relevant executable, supporting libraries and simulation input files in a directory, along with a run. sh crafted to execute it. An example of running Amber this way is shown in Figure 3.

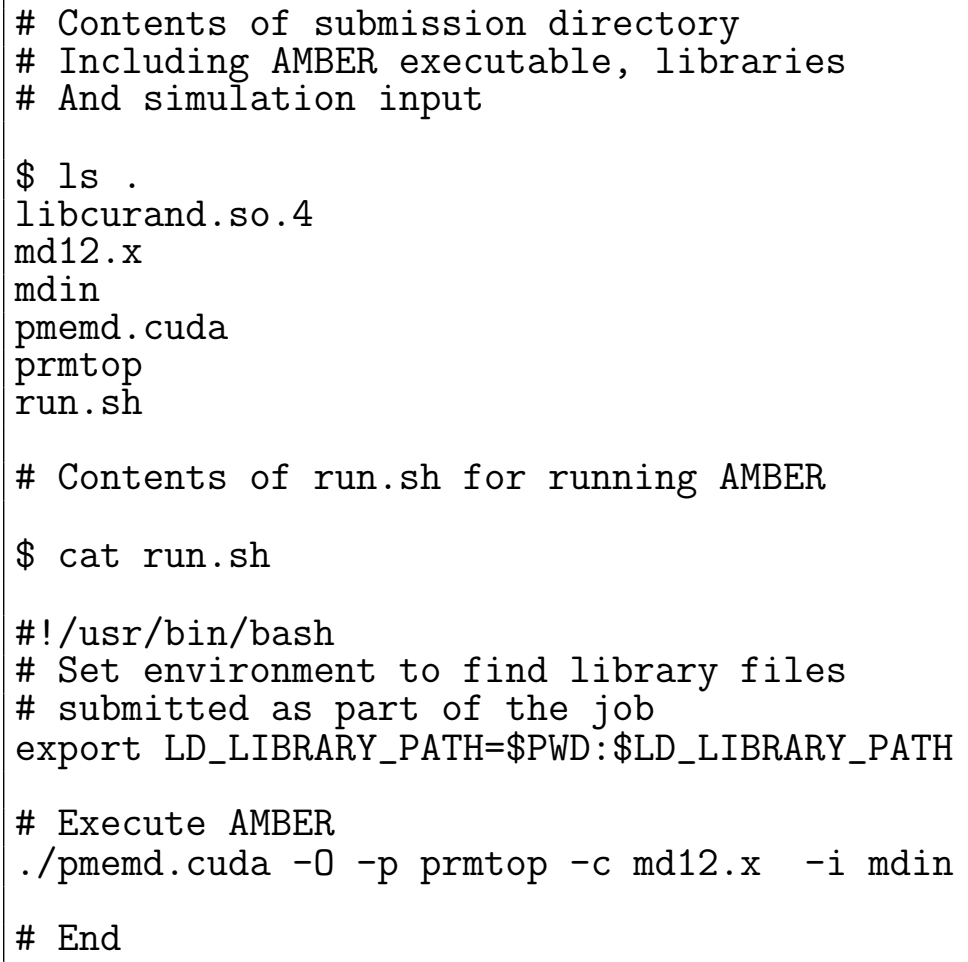

Figure 3: A example of running an arbitrary application via AceCloud, in this case the pmemd program from Amber. ${ }^{3}$ An input directory is prepared containing the pmemd binary itself, along with a supporting library, the input files for the simulation itself and a run.sh script written to run the program.

\section{AceCloud on Amazon Web Services}

AceCloud takes advantage of four major components of Amazon Web Services: the Elastic Compute Cloud (EC2), Simple Storage Service (S3), Virtual Private Cloud (VPC), and Identity and Access Management (IAM)

EC2 provides an Infrastructure-as-a-Service (IaaS) presentation of compute resources. The resource is provided as an instantiation of a virtualised machine (VM) running atop physical hardware, within which the user is free to deploy an arbitrary operating system 
image (known as an Amazon Machine Instance, or AMI).

EC2 provides numerous classes of VM, each representing a different set of resources ( $e g$ number of CPU cores, amount of memory and storage, network connectivity) optimised for particular use cases. Interaction with EC2 for provisioning, monitoring and control of VMs is accomplished manually via an interactive Web console, ${ }^{10}$ or programatically via a web services RESTful API ${ }^{11} .{ }^{12}$

EC2 instances are provisionable on demand, and most frequently billed at fixed hourly rates. Of particular note is the availability of spot pricing. Instances obtained at spot prices are charged at a variable rate that ostensibly ${ }^{13}$ reflects underlying demand. If the spot price rises above the limit set by the procurer when requesting an instance, those instances are liable to be terminated and the resources reallocated to an entity prepared to pay the higher rate. If the user of the instance is able to tolerate this potential inconvenience, spot pricing can be substantially more cost-effective than standard on-demand pricing.

$\mathrm{S} 3$ is an object storage system accessible via a web services interface. Items of data stored within S3 are referenced by a unique key and are treated as read-only, atomic objects. Objects may be grouped into buckets, analogous to filesystem directories, which exist in a namespace global to all AWS users. S3 provides an access control mechanism enabling buckets and objects to be publicly read-writable, or limited in access to appropriately authenticated entities. Where objects are subject to access control, presigned URLs may be created. These contain a cryptographic signature that allow limited read or write access in the absence of credentials.

Although S3 is substantially different to a conventional storage system, ${ }^{14}$ it nevertheless provides (in the case of AceCloud) a useful storage area for simulation state that is persistent and independent of the EC2 instance life-cycle. 


\section{AceCloud Compute Instances}

Within the AceCloud design each user-submitted job is allocated its own instance of the AMI that persists only for the duration of the job. Notably, there is no AceCloud server process: the client interacts only with AWS web service endpoints and instances.

When the AMI is instantiated it boots a Linux operating system image (derived from Amazon Linux) and immediately downloads the job input from S3 via the URL provided in instance metadata and executes the requisite program. Once the run is complete any output generated is stored in S3 via the specified URL and the instance shuts down and is terminated.

Although there is no runtime limit imposed on the instance, there is a limit of $1 \mathrm{~GB}$ for the total size of output data, imposed by the size of the underlying filesystem and object size limits in S3.

The EC2 Instance type currently used by AceCloud is g2.2xlarge, which provides 8 threads of an Intel E5-2670 Xeon CPU, 15GB of RAM and a single Nvidia K520 GPU, based on the compute capability 3.0 GK104 silicon, with 1536 CUDA cores at 800MHz. Performance data is given below.

\section{Security}

Ensuring data security is an important consideration when using external third-party computing resources or transferring data over public networks. AceCloud is designed with a minimal vulnerability surface: VM instances are configured to expose SSH as the only network-visible service and all data movement is performed via HTTPS (to and from S3) or via SSH connections (partial result retrieval). Each AceCloud client install automatically generates its own unique SSH keypair- no third-party SSH access is possible, nor is password authentication permitted.

AceCloud uses the AWS Virtual Private Cloud (VPC) service to provide logical network isolation for EC2 instances. The VPC can be further configured to route all communications 
via a dedicated Virtual Private Network (VPN) connection back to the user's local infras-

tructure. This VPC connection further secures the transmission of potentially sensitive data between client and cloud.

AceCloud requires read-write access to EC2, S3 and VPC services. Rather than assign full access to the user's AWS account, limited credentials can be generated using the Identify and Access Management (IAM) servce. These restrict the client to interacting only with the specified services. Multiple sets of such credentials can be created, allowing several users to independently use a single AWS account (eg for billing purposes).

To provide maximal protection to the Amazon account, EC2 instances never receive any (IAM) access credentials. Instead, pre-signed URLs generated and provided by the client are are used to allow the instance limited access to S3.

For users for whom compliance to industrial or regulatory standards is required, AWS complies to many of the major industry assurance programmes, include SOC1-3, ISO27001 and ISO9001 (A full list is given at ${ }^{15}$ ). AWS services are replicated in several different geographic regions. Users subject to regulatory issues may prefer to restrict their use to resources within certain territories for compliance purposes.

\section{Cost-Optimisation}

As mentioned above, EC2 instances are available with fixed or spot pricing. For the instance type used by AceCloud, the spot price is often as much a 10 times lower than the ondemand price. Historic trend for the spot pricing for the three US regions are given in Figure 4, showing long periods of low pricing interspersed with peaks indicating transient peaks in demand.

Because the price differential between on-demand and spot is so marked, AceCloud is designed to use the latter. Furthermore the client always attempts to allocate jobs to the cheapest zone within the AWS region it has been configured to use. The user may examine the spot pricing across the all AWS regions with the --spots option. Since spot pricing is 
volatile, the user sets a maximum bid price that they are prepared to pay for access. If the spot exceeds that threshold, instances are liable for termination, but costs remain controlled. While the spot remains below the set limit, the user's running instances incur charges at the prevailing spot price for the zone they are running in.

The state of any instances that are terminated is lost. To guard against unnecessary loss of data, the best practice is to periodically perform incremental retrieval of results from long-running or important simulations.

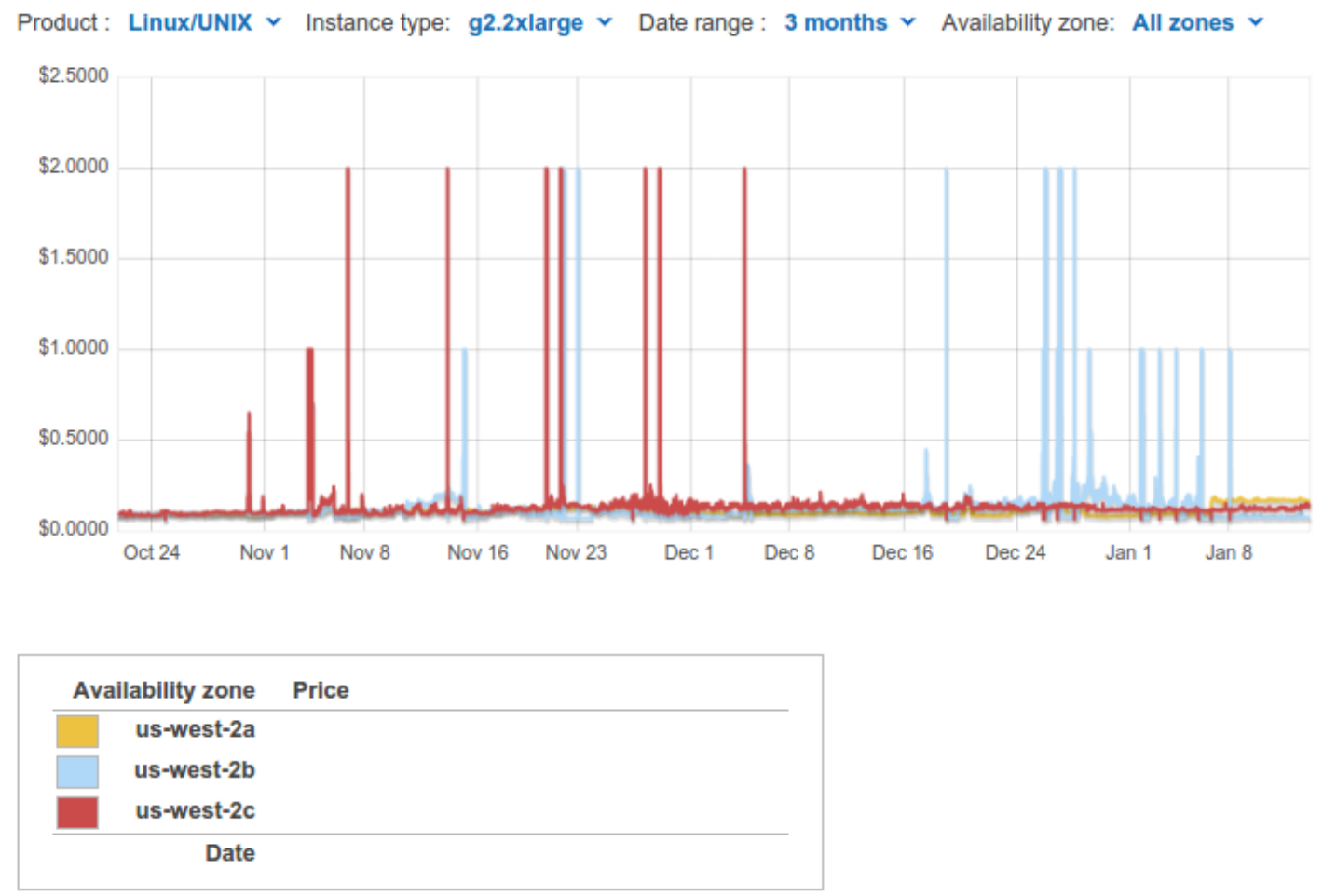

Figure 4: Historic spot pricing over a 3 month period for one AWS region. Pricing is most frequently at a low floor level below the fixed on-demand price, with occasional spikes reflecting peaks in demand (Data and graphs taken from AWS Web Console).

\section{Capabilities and Performance}

AceCloud has built-in support for $\mathrm{ACEMD}^{1}$ and unmodified GROMACS (Version 5.0). ${ }^{2}$ ACEMD supports simulations parameterised with AMBER and CHARMM force fields, running in NVE, NVT or NPT ensembles with the PME treatment of long-range electrostatics. 
Additionally, metadynamics simulations may be performed with PLUMED, ${ }^{16}$ versions 1.3 and 2.0. The AceCloud version of GROMACS supports any simulation type that can be completely specified within a normal TPR-format input file.

The g2.2xlarge EC2 instance type is equipped with an Nvidia K520 GPU. This device is able to attain a rate of $105 n s / d a y$ on the DHFR benchmark (23k atoms, cutoff $=9 \AA, \mathrm{dt}=4 \mathrm{fs}$, PME, NVT). This represents approximately $50 \%$ of the performance of the Tesla K40, the current top-end GPU at the time of writing. Despite the relatively low performance, the cost means they are still economic to use. Furthermore, for ensembles of simulations the overall throughput is of greater importance than that of any one individual simulation (provided that exceeds some acceptable minimum).

The operation of both codes is in no way affected by the virtualised execution environment. Simulation outputs will be equivalent to that produced by execution on local physical resources, subject to the limits of reproducibility for the code in question.

\section{Usage Example}

AceCloud is well-suited to executing ensembles of simulations, such as those employed by De Fabritiis et al in protein-ligand free binding and ab initio protein folding protocols. ${ }^{4,17}$ An example of a 10-way ensemble of the benzamidine-trypsin model system is available for download from. ${ }^{18}$ This section provides a summary of the steps required to execute this ensemble on AceCloud.

Pre-requisites AceCloud access is provided via the current free release of ACEMD, ${ }^{1}$ which should be downloaded and installed. An Amazon Web Services account is also required; these can be created via http://aws . amazon.com/).

AceCloud requires a subscription to the AceCloud Molecular Dynamics Product in the Amazon Web Service Marketplace. The product page is found at https://aws.amazon. com/marketplace/pp/B00Q5ECSOG. Follow the instructions there to subscribe, but do not 
launch any instances.

Finally, the client must be provided with access credentials for the Amazon account. These are obtainable from. ${ }^{19}$ The client is configured with these credentials using the option --configure auth, eg:

\$ acemd --configure auth XXXX-KEY-XXXX XXXX-SECRET-XXXX

Running Simulations Submit each of the 10 simulation inputs using the --submit argument:

\$ acemd --submit --project bentryp *

To monitor the progress, use --status:

\begin{tabular}{|c|c|c|c|c|}
\hline $\begin{array}{l}\text { \$ acemd } \\
\text { Queued } \\
0\end{array}$ & $\begin{array}{r}\text {-status } \\
\text { Running } \\
10\end{array}$ & Completed & $\begin{array}{r}\text { Aborted } \\
0\end{array}$ & $\begin{array}{l}\text { Project } \\
\text { bentryp }\end{array}$ \\
\hline \multicolumn{5}{|c|}{$\$$ acemd --status --long } \\
\hline Project & Statu & & \multirow{2}{*}{\multicolumn{2}{|c|}{$\begin{array}{l}\text { Name } \\
\text { /home/tmp/Benzamidine-Trvosin/o }\end{array}$}} \\
\hline bentryp & RUNNI. & & & \\
\hline bentryp & RUNNI & \multicolumn{3}{|c|}{ /home/tmp/Benzamidine-Trypsin/1 } \\
\hline bentryp & RUNNI & & \multicolumn{2}{|c|}{ /home/tmp/Benzamidine-Trypsin/9 } \\
\hline
\end{tabular}

Jobs will progress from Queued through Running to Completed. Once all jobs are in the Completed state, the output can be retrieved back to the submission directories with --retrieve. To obtain partial output from uncompleted simulations, append the argument --incremental. --verbose can be added to see the operations in progress.

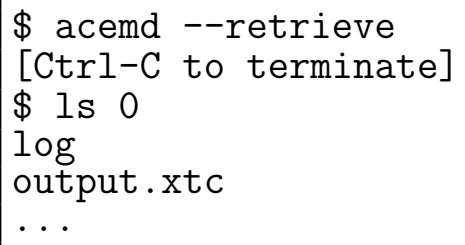

These simulations are each configured to run for 20ns. The compute resources currently used by AceCloud can execute these simulations at a rate of $65 \mathrm{~ns} /$ day, meaning that the ensemble will complete within 8 hours. This throughput exceeds that attainable on a workstation equipped with 4 Tesla K40 GPUs ( $\approx 11 \mathrm{~h})$. 


\section{Discussion}

Molecular dynamics is an established method for in silico investigation of biomolecular processes. In previous work $^{20}$ we have made the case that, as a result of technological and methodological developments, MD is poised for transition from a research context into routine industrial and commercial use.

In logistical terms MD simulation remains challenging to employ: the scale and specialisation of the computing resource required to support state-of-the-art methods ${ }^{17}$ put it beyond casual or occasional use of any group without access to dedicated high performance computing resources. To mitigate this problem, and promote the wider uptake of MD in general, we have developed AceCloud. By exploiting 'cloud computing' - the broad trend in the IT sector towards flexible deployment of compute resources and services through thirdparty providers - we are able to provide low-cost, on-demand access to MD simulation as a robust, production-quality service.

We believe AceCloud will be useful tool for users of molecular dynamics simulation in both industry and academia, and provide a platform for innovation in higher-level protocols, for example adaptive sampling. ${ }^{17}$ Furthermore, AceCloud is an exemplar in delivering access to scientific computing - generally considered the purview of specialised high performance computing centres - through a general-purpose cloud resource provider. The operational benefits that accrue from cloud use are, we contend, substantial and this mode of deployment will become an increasingly common way of delivering resource-intensive computation to end-users across a wide range of scientific disciplines.

\section{Notes and References}

(1) Harvey, M. J.; Giupponi, G.; De Fabritiis, G. ACEMD:Accelerating Biomolecular Dynamics in the Microsecond Time Scale. J. Chem. Theory. Comput. 2009, 5, 1632-1639.

(2) Pronk, S.; Paill, S.; Schulz, R.; Larsson, P.; Bjelkmar, P.; Apostolov, R.; Shirts, M. R.; 
C., S. J.; Kasson, P. M.; van der Spoel, D.; Hess, B.; Lindahl, E. GROMACS 4.5: a highthroughput and highly parallel open source molecular simulation toolkit. Bioinformatics 2013, 29, 1-10.

(3) Salomon-Ferrer, R.; Case, D. A.; Walker, R. C. An overview of the Amber biomolecular simulation package. WIREs Comp. Mol. Sci. 3, 198-210.

(4) Buch, I.; Giorgino, T.; De Fabritiis, G. Complete reconstruction of an enzyme-inhibitor binding process by molecular dynamics simulations. PNAS 2011, 108, 10184-10189.

(5) Buch, I.; Harvey, M. J.; Giorgino, T.; Anderson, D. P.; De Fabritiis, G. HighThroughput All-Atom Molecular Dynamics Simulations Using Distributed Computing. J. Chem. Inf. Model. 2010, 50, 397-403.

(6) Beberg, A. L.; Ensign, D. L.; Jayachandran, G.; Khaliq, S.; Pande, V. S. Folding@Home: Lessons from eight years of volunteer distributed computing. Parallel Distributed Processing, 2009. IPDPS 2009. IEEE International Symposium on. 2009; pp 1-8.

(7) Yates, J.; Hamilton, M.; Dhanoa, H.; Jenner, C.; Hong, N. C.; Hettrick, S.; Parchment, O.; Real, A.; Richards, A.; Burbidge, S. Report of 2014 UK National EInfrastructure Survey (HEIs and Reserch Institutes). 2014.

(8) Armbrust, M.; Fox, A.; Griffith, R.; Joseph, A. D.; Katz, R.; Konwinski, A.; Lee, G.; Patterson, D.; Rabkin, A.; Stoica, I.; Zaharia, M. A View of Cloud Computing. Comm. ACM. 2010, 53, 50-58.

(9) https://aws. amazon.com (Accessed 12 Feb 2015).

(10) https://console. aws. amazon. com (Accessed 12 Feb 2015).

(11) Fielding, R.; Taylor, R. N. Principled Design of the Modern Web Architecture. ACM Trans. Internet Tech. 2002, 2, 115-150. 
(12) AWS Documentation.

(13) Ben-Yehuda, O. A.; Ben-Yehuda, M.; Schuster, A.; Tsafrir, D. Deconstructing Amazon EC2 Spot Instance Pricing. ACM Transactions on Economics and Computation 2013, 1.

(14) The IEEE,; The Open Group, The Open Group Base Specifications Issue 7, IEEE Std 1003.1. http://pubs.opengroup.org/onlinepubs/9699919799/, 2013; [Online; accessed 15 January 2015].

(15) https://aws.amazon. com/compliance/ (Accessed 12 Feb 2015).

(16) Bonomi, M.; Branduardi, D.; Bussi, G.; Camilloni, C.; Provasi, D.; Paitari, P.; Donadio, D.; Marinelli, F.; Pietrucci, F.; Broglia, R.; Parrinello, M. PLUMED: A portable plugin for free-energy calculations with molecular dynamics. Comp. Phys. Commun. 2009, 180, 1961-1972.

(17) Doerr, S.; De Fabritiis, G. On-The-Fly Learning and Sampling of Ligand Binding and High-Throughput Molecular Simulations. J. Chem. Theory. Comput. 2014, 10, 20642069.

(18) Harvey, M. J. ACEMD Simulation Inputs for Benzamidine-Trypsin Ensemble. 2015.

(19) https://console.aws.amazon.com/iam/home?nc2=h_m_sc\#security_credential (Accessed 25 Feb 2015).

(20) Harvey, M. J.; De Fabritiis, G. High-Throughput Molecular Dynamics: The Powerful New Tool For Drug Discovery. Drug. Dis. Today. 2012, 17, 1059-1062. 